\title{
The Development of Topography in the Hamster Geniculo-Cortical Projection
}

\author{
Kristine Krug, Adam L. Smith, and Ian D. Thompson \\ University Laboratory of Physiology, Oxford University, Oxford OX1 3PT, United Kingdom
}

Precise point-to-point connectivity is the basis of ordered maps of the visual field. The immaturity of the newborn hamster's visual system has allowed us to examine emerging topography in the geniculo-cortical projection well before thalamic axons have reached their cortical target, layer IV. Using anterograde transneuronal labeling with wheat germ agglutinin conjugated to horseradish peroxidase (WGA-HRP), we visualized the ingrowth of the whole population of geniculate fibers in the neonatal hamster. Two days after birth (P2), the bulk of the fibers is in the deep cortical layers and the subplate. At the same age, injections of paired retrograde tracers (red and green fluorescent latex microspheres) into area 17 reveal an unordered projection from the dorsal lateral geniculate nucleus (dLGN) to cortex. Individual labeled cells are found throughout the dLGN, and quantitative analysis reveals no segregation of the red and the green populations. At P6, when the pattern of geniculate back label appears ordered and essentially adult-like, geniculate fibers have reached layer IV. The role of selective cell death in this process was investigated by making a tracer injection at P2 and allowing the animals to survive to P6 or P12, when the map is mature. The results show early labeled neurons that made inappropriate connections when the projection was scattered surviving through the period of geniculate cell death. We conclude that the geniculo-cortical map develops from an initially unordered projection to the subplate and the lower cortical layers. Selective cell death appears not to contribute significantly to this process.

Key words: topography; cortical maps; rodent; geniculocortical; map formation; cell death; retrograde tracing; hamster; terminal retraction
Most sensory representations in the mammalian brain are organized topographically, the sensory map reflecting the arrangement of the primary receptor sheet. These maps are a feature not just of the initial relay, but they underpin the structure of sensory areas at different levels throughout the brain. Various developmental mechanisms have been proposed for the creation of the precise point-to-point connectivity that underlies topographic maps. At one extreme, the maps could be sculpted out of an initially random projection by regressive events; at the other extreme, the map in the target may already be laid out in the invading fiber bundle. Studies on the primary retinal projections have demonstrated that both mechanisms can play a role in defining the retinotopic map. Topographic fiber order in the optic tract may contribute to order in the target (Scholes, 1979; Torrealba et al., 1982; Simon and O'Leary, 1991; Reese and Baker, 1993), whereas the retinocollicular map in the rodent, but not in the cat, emerges after a period of axonal rearrangement and ganglion cell death (O'Leary et al., 1986; Simon and O'Leary, 1992; Chalupa et al., 1996). It might be argued that once the map has been generated in the thalamus, a simple relay of fibers to cortex, in which neighbors in the thalamus remain as neighbors in the pathway, will create the cortical map. This is not the case. The orientation of the retinotopic maps in the dorsal lateral geniculate nucleus (dLGN) and in the striate cortex are such that thalamic

\footnotetext{
Received Dec. 18, 1997; revised May 12, 1998; accepted May 15, 1998.
}

K.K. is a Wellcome Trust Prize Student and a Scholar of the Studienstiftung des Deutschen Volkes. A.L.S. holds a Wellcome Trust Career Development Fellowship. This research was also supported by a grant from the Wellcome Trust to I.D.T. We thank Bruce Cumming and Ken Stratford for their invaluable help with the computational analyses, and Patricia Cordery for excellent histological support.

Correspondence should be addressed to Dr. Kristine Krug, University Laboratory of Physiology, Oxford University, Oxford OX1 3PT, UK.

Copyright (C) 1998 Society for Neuroscience $\quad 0270-6474 / 98 / 185766-11 \$ 05.00 / 0$ axons must rearrange on their route to layer IV in cortex (Connelly and Van Essen, 1984; Nelson and LeVay, 1985; Adams et al., 1997).

Exactly where and how this exchange occurs is not known. Work by Agmon and colleagues $(1993,1995)$ on mouse somatosensory cortex shows that at birth, when thalamic axons have already invaded the lower cortical layers, the thalamo-cortical projection is topographically ordered. At this age, the most advanced axons are growing radially through layer $\mathrm{V}$; however, in the deepest cortical layers and in subplate, fibers course tangentially (Agmon et al., 1993) (rat: Catalano et al., 1991, 1996). Such a pattern might imply that the earliest fibers to invade cortex would have a disordered topography. Whether, the disorder is simply local or is the result of the reordering of thalamic fibers that is necessary to generate the cortical map is not clear. We have quantified the topographic organization of the early thalamic input into visual cortex by making paired injections of red and green fluorescent latex microspheres in newborn hamsters. In the hamster, the bulk of thalamic afferents does not enter primary visual cortex until after birth (Crossland and Uchwat, 1982; Miller et al., 1993). Our results indicate massive changes in geniculo-cortical topography in the neonatal hamster. There is little or no topographic organization when the fibers first enter the cortex, but the organization has become adult-like by the time the axons innervate layer IV. By allowing back-labeled cells to survive through the period of map formation, we show that selective cell death makes little contribution to the development of geniculo-cortical topography.

Some of this work has appeared previously in abstract form (Smith et al., 1994; Krug et al., 1995; Krug and Thompson, 1996).

\section{MATERIALS AND METHODS}

Animals. We used hamster pups of time-mated females; the day of birth was counted as postnatal day zero (P0). Some hamster pups had one eye removed on the day of birth under ether anesthesia (for further details, 
see Trevelyan and Thompson, 1992). In these cases, the geniculo-cortical projection contralateral to the remaining eye was examined. This side retains $95 \%$ of its input and has been shown to develop normal adult topography (Trevelyan and Thompson, 1992). For the quantitative measures of topography that we used in this study (nearest neighbor analysis and percentage of dLGN labeled; see below), no significant difference between operated and unoperated hamsters could be detected (two-way ANOVA; $p \leq 0.076$ and $p \leq 0.506$ ), whereas the changes with age for the pooled data were highly significant $(p<0.0005$ in both cases). Therefore, we have pooled the data from unoperated animals with the results from the contralateral side of monocular enucleated animals.

Anterograde tracing. Hamsters at $\mathrm{P} 0$ and $\mathrm{P} 4$ were anesthetized with ether or at P10 with an intraperitoneal injection of a 1:2 dilution of pentobarbitone sodium (Sagatal; RMB Animal Health Limited, Dagenham, UK) $(60 \mathrm{mg} / \mathrm{kg}$ at full concentration) and were given unilateral intraocular injections of WGA-HRP (4\% in sterile saline containing 0.02\% Fast Green FCF: 150-500 nl). After $48 \mathrm{hr}$ survival (except for a single animal at P1 when a $24 \mathrm{hr}$ survival was used), they were perfused with PBS followed by a mixture of $1 \%$ paraformaldehyde/ $1.25 \%$ glutaraldehyde in $0.1 \mathrm{M}$ phosphate buffer followed by PBS. After cryoprotection with $30 \%$ sucrose, the brain was cut parasagittally at $50 \mu \mathrm{m}$ intervals. Slide-mounted sections were reacted with tetramethylbenzidine by a modified standard procedure (Mesulam, 1982), and adjacent sections were stained with cresyl violet.

Retrograde tracing. At certain postnatal ages (P2, P4, P6, or P12), anesthetized hamsters received paired injections of red and green fluorescent latex microspheres (diluted 1:10; LumaFluor, Naples, Florida) into area 17. For neonatal surgery up to P6, hamsters were anesthetized with a dilute solution (1:10) of alphaxalone $0.9 \%$ and alphadolone acetate $0.3 \%$ (Saffan, Pitman-Moore, Uxbridge, UK) (intramuscularly, equivalent dose of $0.5 \mathrm{ml} / \mathrm{kg}$ at full concentration). Older animals (P12) were injected intraperitoneally with a 1:2 dilution of pentobarbitone sodium (Sagatal, as above). The scalp was resected, and discrete placements of the retrograde tracer were made perpendicular to the cortical surface either through pin pricks in the skull at ages P2-P6 or after craniotomy at P12. Injections were spaced mediolaterally, 300-800 $\mu \mathrm{m}$ apart; the anteroposterior position was roughly level with $\lambda$. According to age, between 25 and $100 \mathrm{nl}$ of tracer was injected through glass pipettes (inside tip diameter 10-25 $\mu \mathrm{m}$ ) under air pressure. Except for the P12 animals, injections usually involved both cortical plate and subplate. The scalp was resutured, and the animal was allowed to recover in an incubation chamber. Hamster pups (2- and 4-d-old) were killed after 12-24 hr with an overdose of pentobarbitone sodium. Animals 6-d-old and older were returned with their litter mates after recovery; survival times were $24 \mathrm{hr}$ for P6 injections and $48 \mathrm{hr}$ for P12 injections. Animals were perfused through the heart with PBS followed by $4 \%$ paraformaldehyde in $0.1 \mathrm{M}$ phosphate buffer.

For the heterochronic experiments, a specific group of animals were injected with only one tracer at $\mathrm{P} 2$, and after recovery they were put back with their litter mates. Subsequently, either at P6 or at P12, the animals were reanesthetized, the skull was exposed, and the position of the previous tracer placement was identified under UV light through the appropriate filter. We marked the location, made a hole using a pin prick, after drilling, if necessary, and a differently colored tracer was injected. After survival times of 24 (P6) or $48 \mathrm{hr}$ (P12), the animals were overdosed with pentobarbitone sodium and perfused with PBS and 4\% paraformaldehyde as above.

After post-fixation, brains were removed from the skull and soaked in $30 \%$ sucrose until they sank. Then coronal sections were taken at $50 \mu \mathrm{m}$ on a freezing microtome. One in two series were mounted on gelatinized slides. One series was coverslipped using Fluoromount medium, and the other was stained with cresyl violet and coverslipped with DPX.

The size of cortical injection sites was estimated by aligning measurements of their mediolateral extent in layer IV from all coronal sections on which the sites were visible. The distance between the individual sections in a series was $100 \mu \mathrm{m}$. The resulting area was measured with a scanning program (SigmaScan, Version 3.90 for DOS; Jandel Scientific, Corte Madera, CA). The extent of area 17 was taken from the thalamorecipient zone in animals transneuronally labeled after intraocular injections with WGA-HRP at the corresponding ages (see above and Results). Thus, we could represent the size of our injection sites in the percentage of area 17 labeled.

Criteria for cell sampling. Geniculate neurons retrogradely labeled with fluorescent tracers were sampled with a computer program. Using a Zeiss fluorescent microscope, a camera lucida view of the display screen was optically superimposed onto the section. The outlines of the dLGN were drawn into the computer at low magnification $(2.5 \times$ objective $)$, and a sample grid $(200 \times 200 \mu \mathrm{m})$ was superimposed. Labeled cells were drawn at a higher magnification $(25 \times$ objective) using a $200 \times 200 \mu \mathrm{m}$ sample box, thus ensuring that all the labeled cells within the dLGN were counted. Only cells in which the retrograde tracer defined the cell soma were drawn. For each sample box, the locations of all cells of one color were marked first. Then the display screen was cleared, and the filter set was changed so that the population of cells labeled with the second tracer could be drawn independently. The computer package then identifies as candidates for double-labeled cells red and green profiles whose centers were less than 10 pixels $(5.8 \mu \mathrm{m})$ apart. These were subsequently assessed visually and classified as double-or single-labeled. The dLGN borders were verified from the Nissl-stained sections. The program contains information about the absolute locations of cells labeled by the two tracers and the positions of dLGN borders. These data were then imported into SYSTAT (for DOS or Version 5 for Windows; SYSTAT, Evanston, Illinois) for visualization and printouts.

Analysis. Our analysis was performed in the coronal plane. In the adult hamster, a column running roughly rostrocaudally feeds into one point in cortex (Dursteler et al., 1979), and mediolaterally spaced injections in cortex give labeled foci that are separate in the coronal plane. Coronal sections were taken through the whole dLGN, and most of the refinement we observed after an injection could be seen in individual sections. The order in the geniculo-cortical projection was quantified in three ways. The extent of dLGN labeled by a single injection was estimated with isodensity contours. A program written in "C," calculated local densities (cells $\mathrm{mm}^{-2}$ ) by convolving the $x-y$ array of one population of cells in each section with a two-dimensional Gaussian $(\mathrm{SD}=21 \mu \mathrm{m})$. The local density was evaluated in $6 \mu \mathrm{m}$ steps across the dLGN, and then the peak density for each type of label across all sections in a single dLGN was determined. Finally, the program calculated the area that contained label $\geq 10 \%$ of the peak density. The results across all sections of a given dLGN were summed, and the sum was divided by the total area of the sampled dLGN sections.

A nearest neighbor analysis estimated the degree of segregation between two populations of labeled cells by determining the probability that a given labeled cell has as its nearest neighbor a cell of the same color. A Pascal Program ran through each population of labeled cells and noted whether any one labeled cell had as its nearest neighbor a cell of the same ("1") or a different color (" 0 "). These values were summed and averaged separately for the red and green populations in each section. If the two populations were totally segregated a value of 1.0 would be expected, but if the two groups were distributed at random and of the same size (see below), the value would be 0.5 . Because the individual sections contained different numbers of labeled cells, the neighbor values for each section were weighted according to the relative number of red or green cells on that section. The weighting was done separately for the red and green populations to account for the possibility that segregation was occurring not in the plane of the section but across sections. This gave two neighbor values for the whole dLGN, one for the red population and one for the green population. These two values were then averaged to give a final single neighbor value for each dLGN, which was expressed as a percentage. The reason for this final average is because the neighbor measure is sensitive to imbalances in the total numbers of red and green cells. For instance, even if the two populations are truly randomly segregated, the neighbor value of the smaller population would deviate from 0.5 toward 0 as the relative number of cells in that population declines. Similarly, the value for the larger group would approach 1.0 as it became relatively more numerous. Simulations of the effects of population size differences for different degrees of segregation revealed that there was a symmetrical deviation in neighbor values for the two groups from the value seen when the two groups were matched in size. Thus, the increase in the nearest neighbor value of the larger population mirrors the decrease in the smaller one, for up to a 1:4 ratio in relative numbers. Consequently, animals in which the ratio of total red and green cell numbers in the dLGN was less than 1:4 were not used. The symmetric deviation allowed us to average the separate red and green nearest neighbor values to give a final nearest neighbor value for each analyzed dLGN. To be able to compare nearest neighbor values across the different ages studied, we used similar injection site separations in all animals. Since the mediolateral extent of area 17 expands in the first 2 postnatal weeks (Table 1), the relative cortical spacing of the injection sites is larger in the younger animals, which might bias the early results toward a more segregated value. 
Finally, the spatial relationship between the two foci of labeled cells on each section was determined by calculating the vector between their respective centers of gravity. In mature animals, this relationship is stable for a given injection site pattern. First the orientations of sections were aligned to match the dorsoventral and mediolateral axes. The $x, y$ coordinates of each cell were known. Sections with fewer than 10 cells in either population were excluded from the analysis. Then the mean $x$ and $y$ values were calculated for the red and the green population in each section. The origin of the vector was always taken as the center of gravity of the population of cells labeled from the more medial cortical injection. In the adult, such an injection always labels a more lateral population of cells, but this relationship was not always evident in younger animals. This strategy was adopted to determine whether a bias toward the adult map can be revealed in the early highly scattered projection.

\section{RESULTS}

\section{The bulk of the transneuronally labeled geniculate projection enters layer IV after P2}

The distribution of the geniculate afferents in cortex was revealed using transneuronal transport of WGA-HRP. Intraocular injections gave continuous labeling in the contralateral dLGN in animals (except at P12, when the crossed and uncrossed inputs have segregated). The labeling in cortex thus reveals the total distribution of geniculate fibers rather than the trajectory of a small number of axons. Examples of the transneuronal labeling seen in parasagittal sections after injections at different ages are shown in Figure 1, together with the corresponding Nissl-stained sections. Figure $1 A$ illustrates the projection seen $48 \mathrm{hr}$ after an injection at P0. While the fibers show a regionally restricted arborization in posterior cortex, the bulk of the label is confined to the subplate and the deeper cortical layers. In all five animals surviving for $2 \mathrm{~d}$ after injection at P0, label was observed throughout the subplate. Some labeling of the cortical layers was seen in four animals, and in two of these this extended to the dense cortical plate, which contains the recently migrated upper layer $\mathrm{V}$ and lower layer IV neurons (Crossland and Uchwat, 1982; Miller et al., 1993). A single animal surviving only $24 \mathrm{hr}$ until P1 displayed very restricted transneuronal labeling in subplate.

After injections at P4 and $48 \mathrm{hr}$ survival to P6, the distribution

\section{Table 1. Measurements of area 17}

\begin{tabular}{llll} 
& $n$ & Area $\left(\mathrm{mm}^{2}\right)$ & $\begin{array}{l}\text { Mediolateral } \\
\text { extent }(\mathrm{mm})\end{array}$ \\
\hline P2 & 5 & $2.76 \pm 0.67$ & $1.58 \pm 0.31$ \\
P6 & 5 & $4.27 \pm 0.95$ & $1.80 \pm 0.21$ \\
P12 & 4 & $5.55 \pm 0.74$ & $2.20 \pm 0.23$
\end{tabular}

The table contains average measurements and their SDs of the area and the maximum mediolateral extent of area 17 at various postnatal ages. The area was determined by transneuronal labeling of thalamic afferents and represents the major projection zone. of thalamic axons in the cortex is very different (Fig. $1 B$ ). The bulk of the label is now remote from the fasciculated projection deep to the subplate and is found in layer IV, just below the dense cortical plate. By P12 (Fig. 1C), the superficial layers have migrated into position, and the distribution of geniculate fibers is essentially adult-like, with most label being confined to layer IV. At all ages the extent of the transneuronal labeling in parasagittal sections shows clear anterior and posterior restrictions (Fig. 1). This has allowed us to define area 17 in animals of different ages and to measure changes in its extent (Table 1). The mean area of primary visual cortex doubles between P2 and P12, and growth appears to be symmetric in both the anteroposterior and mediolateral axes.

\section{Retrograde labeling of the geniculo-cortical projection by paired injections visualizes the emergence of topography}

Having defined the cortical location of the geniculo-cortical terminals with transneuronal labeling, we made paired injections of red and green latex microspheres to determine the topographic organization of the projection. Injections were placed perpendicular to the cortical surface, and the injection sites included cortical plate and subplate in the younger animals; only at P12 was it possible to restrict the tracer placements systematically to the cortical plate. The injections were separated mediolaterally. Similar ranges $(300-800 \mu \mathrm{m})$ were used at all ages, and the mean separations are comparable (Table 2). However, it should be noted that area 17 has a lower mediolateral extent in the younger animals (Table 1), so that the relative mediolateral separation is wider in these neonates.

\section{At P2, the geniculo-cortical projection is scattered}

Two discrete injections of red and green tracer into area 17 at P2 retrogradely label cell bodies throughout the ipsilateral dLGN with considerable intermingling of red- and green-labeled cells on any one coronal section (Fig. 2). Inspection of Figure 2 shows that labeled cells of a single color can be found throughout the entire dLGN. We have quantified the spread of retrograde labeling by measuring the area of each coronal section that contains labeled cells at a density of at least $10 \%$ of the peak density seen in that dLGN (see Materials and Methods). Nearly three-quarters of the dLGN is labeled at this or a higher density of label (see below).

Although labeled from separate cortical sites, the red and the green population of cells appear congruent in the P2 dLGN, an observation confirmed by nearest neighbor analysis (see below). Only a small percentage of the neurons is back-labeled by both tracers (16\% at P2) (Table 2). This eliminates the possibility that the spread of retrograde label seen in Figure 2 reflects a greater spread of tracer across the cortex than was apparent from the

Table 2. Summary data

\begin{tabular}{|c|c|c|c|c|c|c|}
\hline & $n$ & $\begin{array}{l}\text { Injections: } \\
\text { mediolateral } \\
\text { separation }(\mu \mathrm{m})\end{array}$ & $\begin{array}{l}\text { Injection } \\
\text { size in \% } \\
\text { area } 17\end{array}$ & $\begin{array}{l}\text { Double- } \\
\text { label }(\%)\end{array}$ & $\begin{array}{l}\text { Percentage of } \\
\text { dLGN labeled }\end{array}$ & $\begin{array}{l}\text { Percentage } \\
\text { of nearest } \\
\text { neighbor same }\end{array}$ \\
\hline $\mathrm{P} 2$ & 7 & $575 \pm 160.5$ & $1.66 \pm 1.2$ & $16 \pm 6$ & $73 \pm 7.6$ & $48.8 \pm 4.62$ \\
\hline $\mathrm{P} 4$ & 3 & $550 \pm 40.5$ & & $7 \pm 3$ & $38 \pm 5.2$ & $66.7 \pm 14.23$ \\
\hline P6 & 8 & $481 \pm 185$ & $1.29 \pm 0.78$ & $8 \pm 6$ & $19 \pm 8.0$ & $80.8 \pm 7.38$ \\
\hline $\mathrm{P} 12$ & 7 & $557 \pm 203$ & $1.27 \pm 0.47$ & $6 \pm 7$ & $13 \pm 8.1$ & $90.6 \pm 5.09$ \\
\hline
\end{tabular}

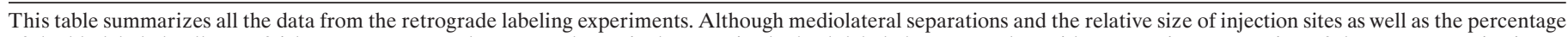

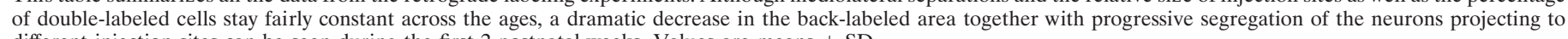
different injection sites can be seen during the first 2 postnatal weeks. Values are means \pm SD. 

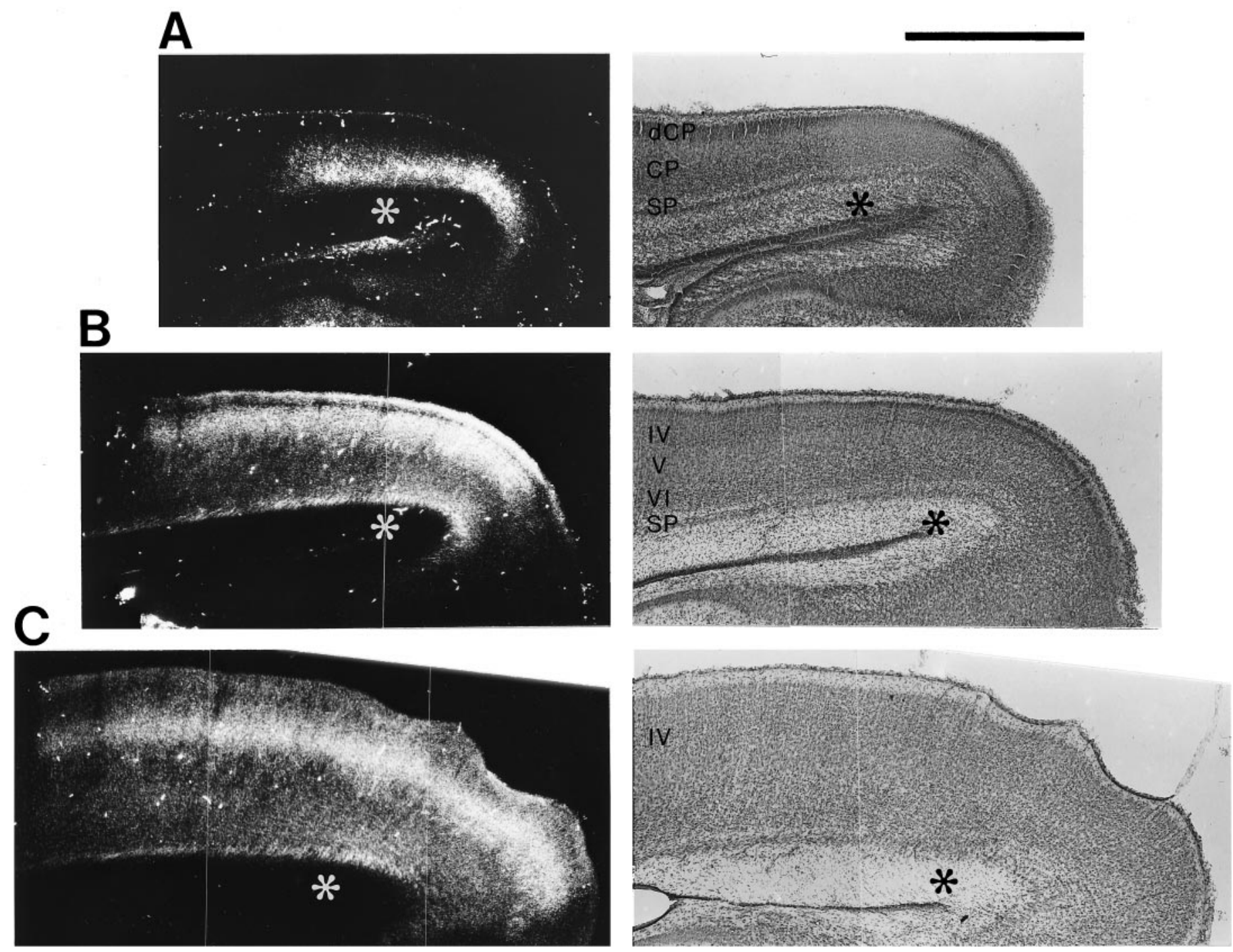

Figure 1. Anterograde tracing of the geniculo-cortical projection. Transneuronal labeling of geniculate axon terminals with intraocularly injected WGA-HRP was used to monitor the development of geniculo-cortical connectivity. Dark-field photomicrographs, taken with cross-polarizers, of WGA-HRP reaction product in parasagittal sections are shown for $\mathrm{P} 2(A), \mathrm{P} 6(B)$, and $\mathrm{P} 12(C)$ on the left-hand side, with corresponding sections stained for Nissl substance on the right-hand side. $A$, Geniculate axon terminals are found in the subplate and cortical plate at $\mathrm{P} 2$, but have not yet invaded the dense cortical plate that is to become layer IV. B, By P6 the majority of fibers are found within cortical layer IV, although some label can still be seen deeper. The periodic nature of the label at the border between subplate and white matter indicates fiber fascicles entering cortex. $C$, At P12, we see an adult-like distribution of label. By this time the superficial layers have migrated into position and below these the label is largely confined to layer IV. As in P6 animals, the label at the subplate/white matter interface represents fiber fascicles. Asterisks mark corresponding portions of the intermediate zone on the pairs of photomicrographs. Scale bar, $1 \mathrm{~mm}$. $C P$, Cortical plate; $d C P$, dense cortical plate; $S P$, subplate.

injection site. On visual inspection, injection sites were discrete and nonoverlapping. Reconstruction of injection sites at P2 shows that each occupies $<2 \%$ of the cortical area at this age (Table 2). For undetected spread alone to account for the extensive geniculate labeling, the effective injection sites would have to be much larger. The existing injections are 100-250 $\mu \mathrm{m}$ in diameter, so any substantial increase would generate overlap and increased double labeling. Most cells are not double-labeled, and the doublelabeled cells are scattered throughout the dLGN.

Uptake by fibers of passage also cannot explain the diffuse pattern of back label. After multiple injections of beads into cortical plate and subplate anterior to area 17 , retrogradely labeled cells were restricted to the ventrobasal complex. This is illustrated in the left-hand panels of Figure 3. The green beads were injected deep to assess any labeling of fibers of passage running to more caudal cortical areas such as visual cortex. Green beads were not seen in dLGN neurons, but back-labeled cells were found throughout the ventrobasal complex. In this animal, a single small red injection was made into visual cortex (not shown); neurons labeled with red beads are found throughout dLGN. In older animals, it is easier to restrict bead injections to the upper cortical layers, but injections that also invade the lower layers and subplate give the same pattern of geniculate back label. In the right-hand panels of Figure 3, two similar foci of red- and greenlabeled cells can be seen in the dLGN, although the red injection involved the deeper cortical layers and subplate. Thus injections of beads made through micropipettes are not taken up significantly by geniculate fibers of passage deep in visual cortex. This 


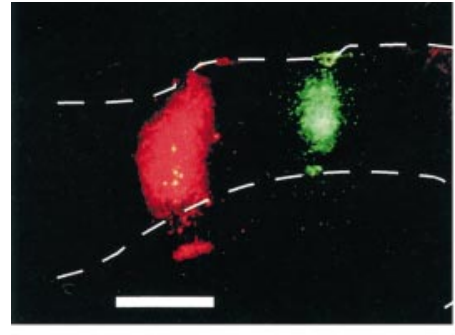

P2
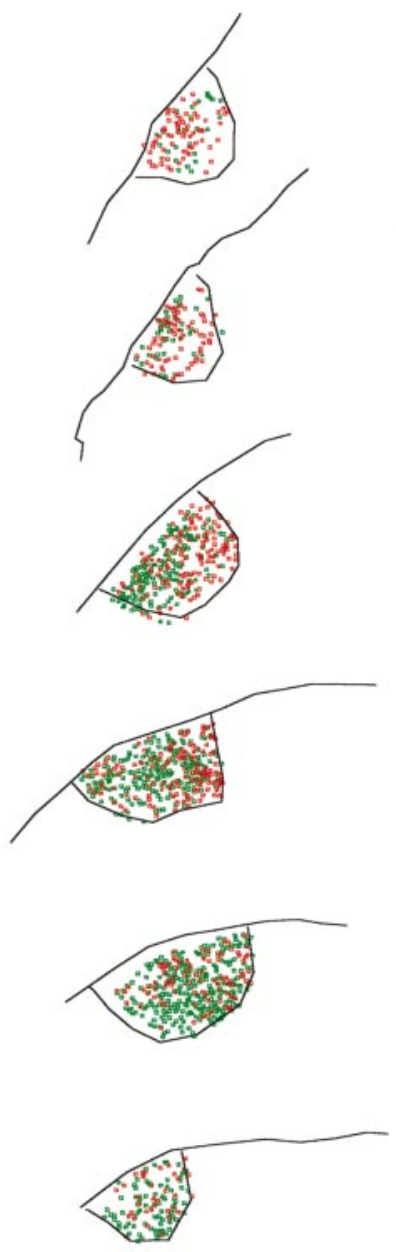

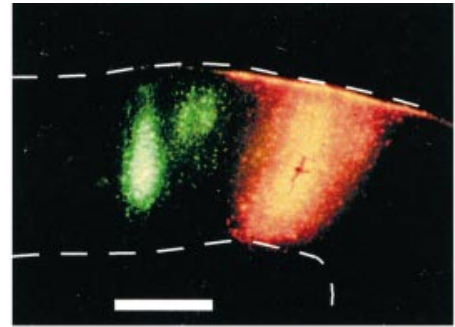

P6

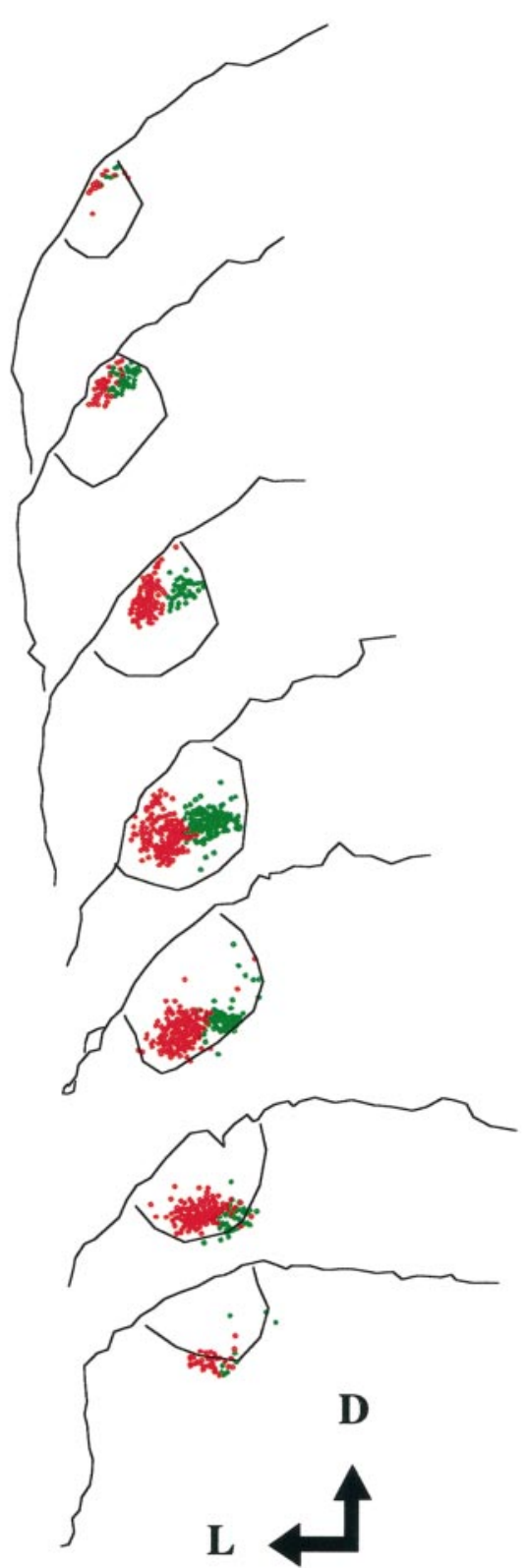

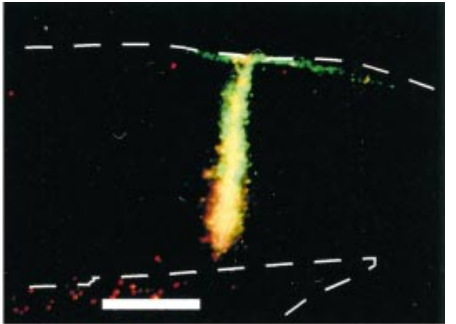

P2 to P12

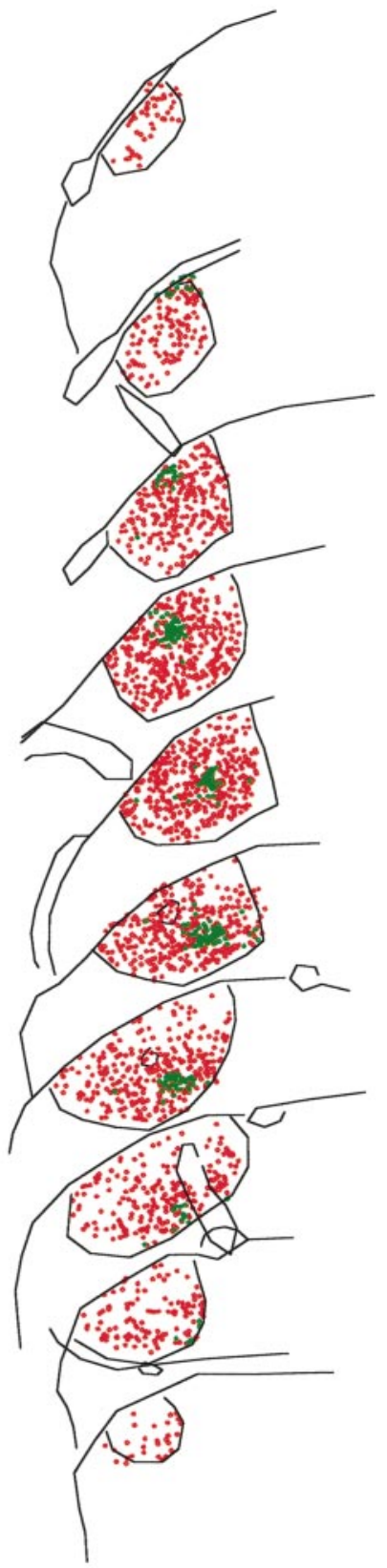

Figure 2. The pattern of geniculate back label during the first 2 postnatal weeks. The top of the figure shows cortical injections of the red and green fluorescent tracers, seen in coronal sections. The white dashed contours mark the pial surface and the border between subplate and white matter. Scale bars, $500 \mu \mathrm{m}$. Underneath are representations of series of coronal sections through the dLGN (50- $\mu \mathrm{m}$-thick, one in two series). The sections run caudorostrally from top to bottom. Each colored dot indicates the location of a neuron back-labeled with either the red or the green tracer. The sections on the left depict the pattern of back label as generated by paired mediolaterally spaced injections at P2. Two days after birth (Figure legend continues), 
confirms the observations of Trevelyan (1992) in adult hamsters: placements of red and green tracers along the same pipette track, one into the cortex and the other into the white matter, yield back label in the dLGN almost exclusively from the cortical injection.

\section{By the end of the first postnatal week, the projection} appears ordered

Cortical injections were made at P4, P6, and P12. Inspection of the pattern of labeling reveals that clear geniculo-cortical topography has emerged by the end of the first postnatal week. After injections on P4, although labeled cells of both colors are widely scattered across the dLGN, distinct condensations of red or green cell bodies can be discerned. At P6, the pattern of back label looks essentially adult-like. On coronal sections, two discrete foci of labeled cells are visible, one red and one green (Fig. 2). These foci appear dorsomedially on the more caudal sections and move through the dLGN to the ventrolateral margin in more rostral sections, thus forming an ordered column through the nucleus. The columns of red- and green-labeled cells are spatially separate, with very little overlap. In Figure 2, the more medial column of green cells arose from a cortical injection of green beads placed laterally to the red tracer. The topography is that expected from the adult. Qualitatively, only small changes seem to take place over the following week: P6 and P12 injections yield a similar pattern of back label.

\section{Quantitative analysis of the developing geniculo-cortical projection}

With a single tracer, it is possible to measure the extent of the dLGN occupied by retrogradely labeled cells using a density analysis (see Materials and Methods). Having used two tracers, we were also able to measure both the percentage of doublelabeled cells (indicating the spread of individual axonal arbors) and the nearest neighbor relations of the two populations (giving a measure of the spatial segregation of the two groups of cells).

Looking at the changes in percentage area of dLGN labeled, we get an estimate of the convergence in the projection onto one point in cortex. Our criterion of density ensures that we assess only the area of dLGN that contains a certain baseline of label. Approximately $70 \%$ of the dLGN is labeled at or above $10 \%$ of the peak labeling density after a single injection at $\mathrm{P} 2$ (Fig. $4 A$ ). This value drops to approximately less than one-quarter when injections are made at $\mathrm{P} 6$, although the relative injection sizes remain between 1.66 and $1.29 \%$ (Table 2). Relatively little change with age is seen in the percentage of geniculate cells that are double-labeled (Fig. 4C). The small drop in the percentage of double-labeled cells we see between P2 and older animals could reflect changes in relative size of the injection (Table 2) or changes in the number of collateral branches (see Discussion). At the same time, the probability that a cell has a nearest neighbor of the same color increases. At P2, a labeled neuron is as likely to have as its nearest neighbor a cell filled by the same or a different tracer (Fig. 4B). This suggests a random distribution of label; the input to the two discrete injection sites is not segregated in the dLGN. The nearest neighbor value rises from approximately chance at $\mathrm{P} 2$ to $\sim 80 \%$ at $\mathrm{P} 6$; the input to different points in cortex segregates (Fig. 4B). Between P6 and P12, the figures for the nearest neighbor analysis and the percentage of dLGN labeled reflect further refinement ( $t$ test, $p<0.05$ ), but on a smaller scale than before.

The nearest neighbor results suggest that the early projection to area 17 is random. To test whether there is any bias toward the adult columns in the early scattered label, we calculated the vector from the center of the population of cells labeled by the medial injection to the center of the population labeled by the lateral injection. This was done for every section (Fig. 5). The size of the vector indicates the degree of segregation of the two clusters of label, and its direction should be predictable from the adult pattern of columns: a medial injection labels cells laterally in the dLGN and vice versa. The graph shows that the two foci of label segregate more and more during the first 2 postnatal weeks. At P4 and later almost all vectors point to the right, i.e., medially. Because of the alignment of the projection columns (Fig. 2), there is also a small dorsoventral component to the vector. At P2, however, the vectors cluster around zero, and some sections in all animals yield vectors that point laterally. Although there are sections with the correct bias in the pattern of label, there seems to be no overall bias for the adult-like columnar organization. Thus, these measurements underline the picture of a random geniculate projection to the subplate and lower cortical layers at $\mathrm{P} 2$. At the later ages, the increase of the vector length between the two clusters, in conjunction with the reduction in the area of dLGN back-labeled, indicates greater segregation of the input to the two injection sites.

\section{Retrograde labeling of geniculate projection neurons by heterochronic injections suggests little role for cell death in map formation}

To reveal what part the selective death of inappropriately projecting geniculate neurons might play in the development of an ordered projection, we injected one tracer into area 17 at P2, before topography is established, and allowed the animal to survive through the maturation of the geniculo-cortical map to P6 or P12. At either of these ages, an injection of the second tracer was placed into the same cortical site as the P2 injection. If selective cell death is the major mechanism sculpting the adult map from the early scattered projection, with two spatially superimposed heterochronic injections we should see two congruent populations of labeled geniculate cells, because all cells that had originally made inappropriate projections would have been eliminated. In contrast, any neurons that are labeled by the early injection but are outside the area labeled by the later tracer placement have survived, despite making an initial projection to a topographically incorrect target.

Figure 2 (right) shows that the pattern of geniculate labeling seen after long-term survival after a P2 injection does not differ qualitatively from the $\mathrm{P} 2$ result seen after $12-18 \mathrm{hr}$ survival. The red injection labels cell bodies scattered throughout the dLGN. The somas were clearly defined by the beads. At an age when the geniculo-cortical connectivity has reached an adult-like appear-

\footnotetext{
the distribution of the retrograde label is diffuse; in every section there are red cells intermingled with green cells and vice versa. The middle row of sections stem from an animal injected at P6 and show an ordered map. The lateral, green cortical injection produces a distinct medial focus of labeled cells, whereas the medial, red injection back labels an adjacent lateral focus of cells. The sections on the right come from an animal in which rhodamine beads were injected at P2, and at P12 green beads were placed at the same cortical site (in the double-exposure photomicrograph the spatially coincident injections appear yellow). The cells labeled with green form a tight focus moving through the nucleus, whereas the early labeled red cells remain diff usely distributed across the dLGN, not merely colocalized with the green cells.
} 
P2
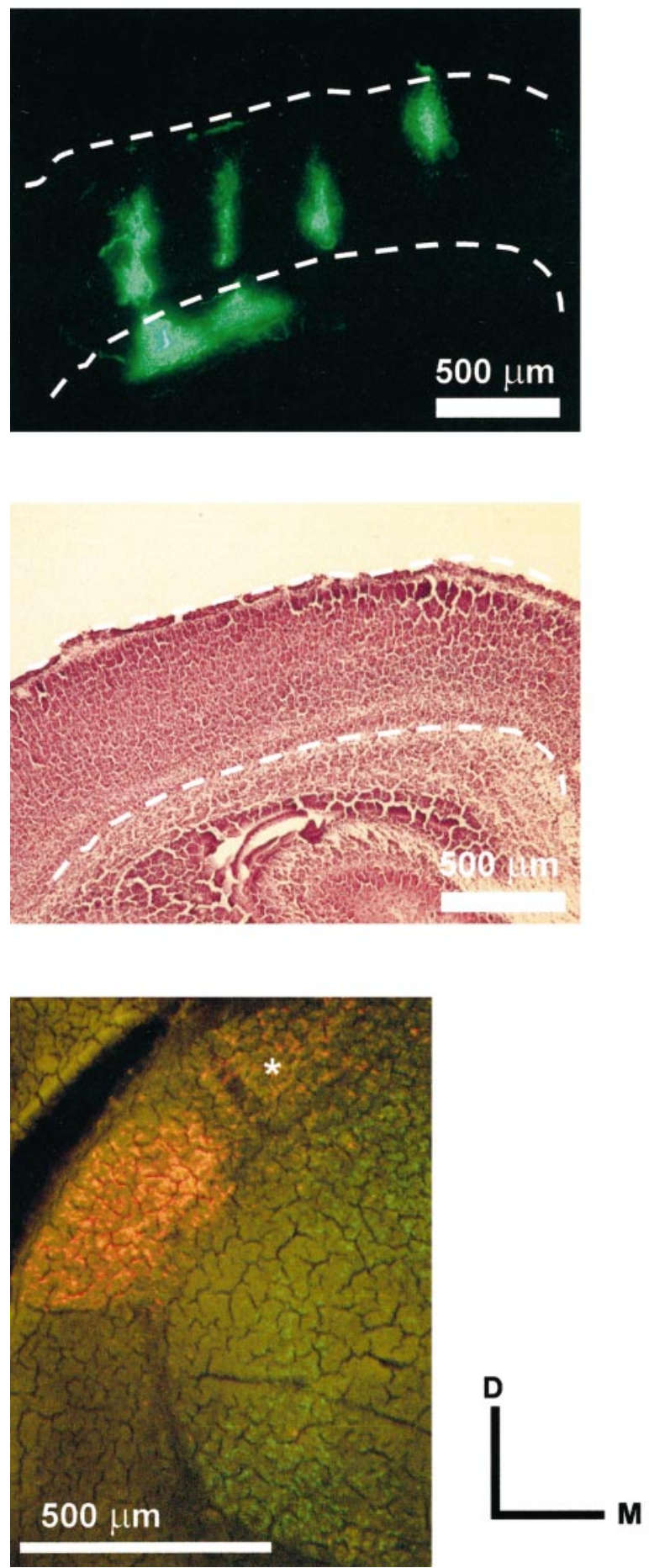

P6
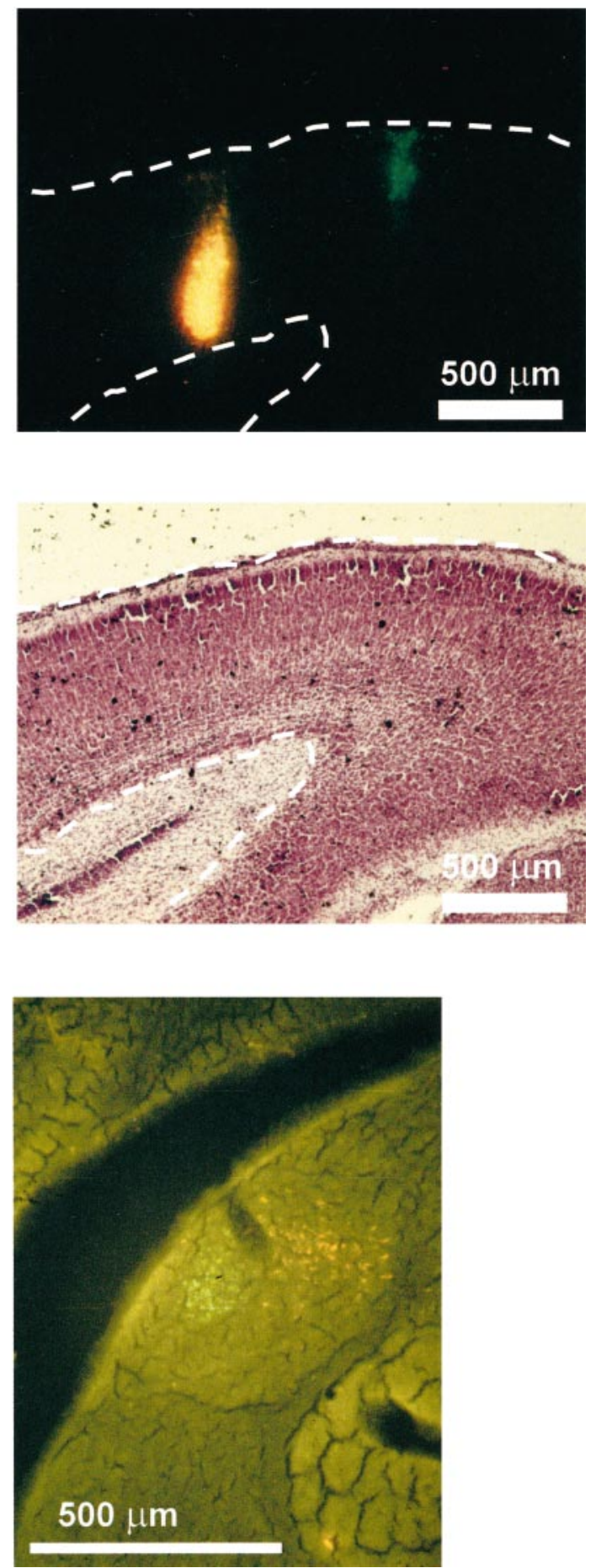

Figure 3. Controls for uptake by fibers of passage. These photomicrographs of coronal sections through dLGN and cortex depict control experiments to test whether fibers of passage in developing cortex take up the fluorescent microspheres. The three panels on the left come from a P2 hamster. A single injection of red beads was placed as normal in visual cortex (injection not shown), and multiple injections of green beads were made in a mediolateral row approximately $1.5 \mathrm{~mm}$ rostral to the red beads. The injection sites are shown under fluorescence illumination in the top panel, and the adjacent Nissl section shows that the injections penetrated the cortical plate, the subplate, and the marginal zone. After such large injections, which should involve geniculate axons running caudally to area 17, the dLGN contained less than a handful of green cells, although many green back-labeled cells can be seen in the ventrobasal complex. As expected, many labeled cells can be seen in the dLGN and lateral posterior nucleus (LP) after the single injection of red beads. The three panels on the right come from a hamster in which injections were made at P6. In this animal, a small green injection was made medially; comparison of the injection site and the Nissl-stained section shows that it did not involve the deeper cortical layers. The larger, more lateral red injection involved both superficial and deep cortical layers. The bottom panel shows red and green labeled neurons in the dLGN, both in discrete foci. $D$, Dorsal; $M$, medial; *, LP. 
A. Percentage dLGN Labeled

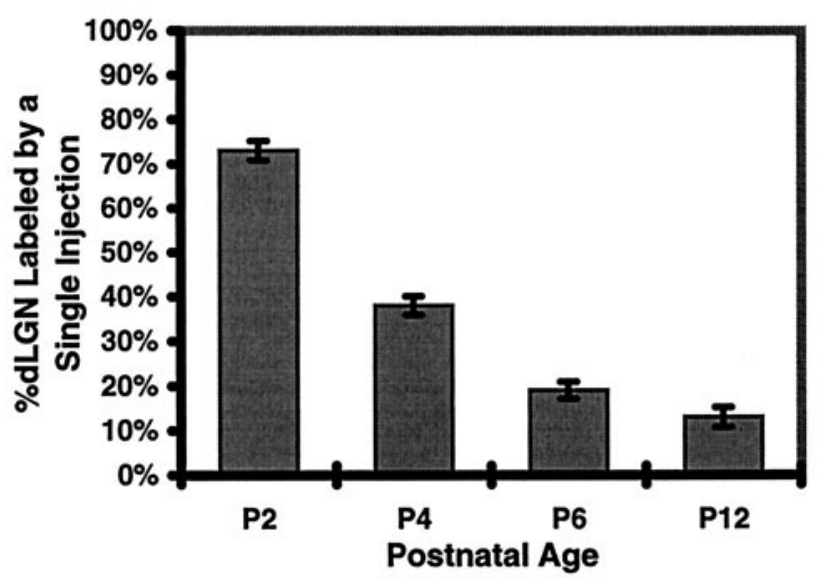

B. Nearest Neighbour Analysis

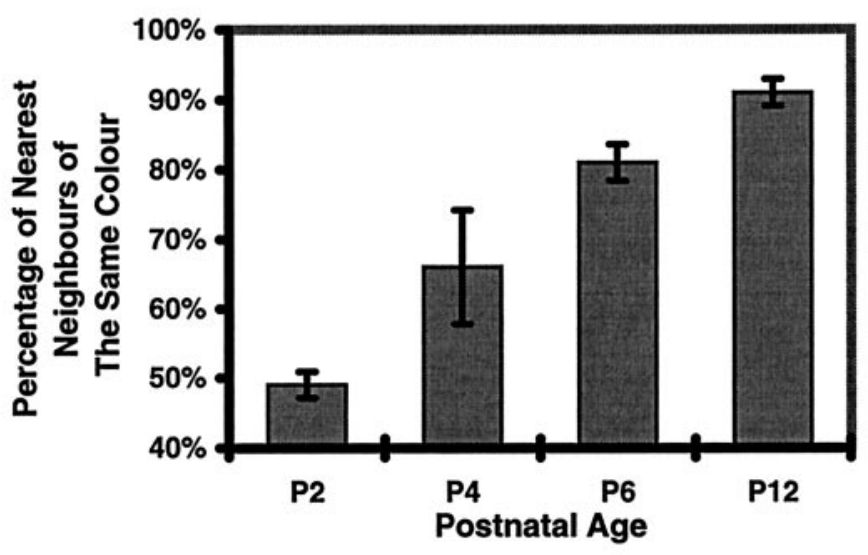

C. Percentage Double Label

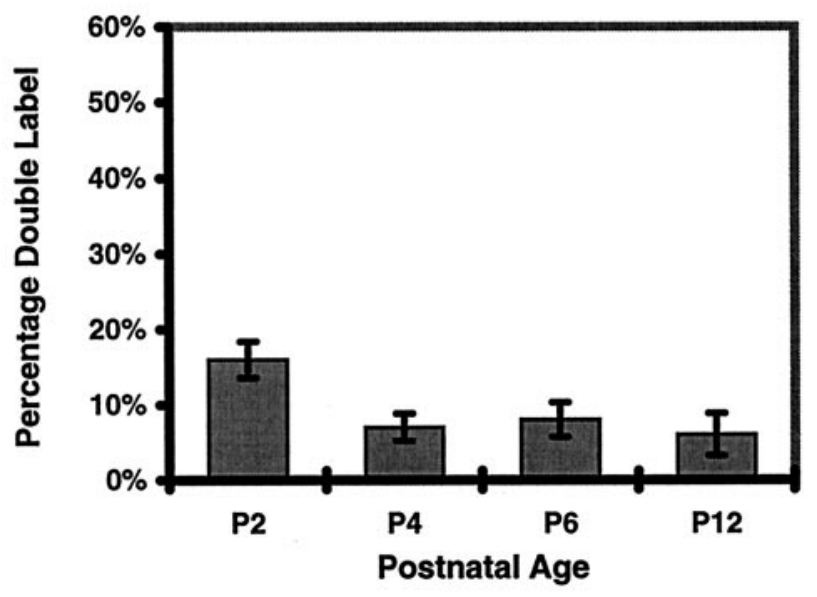

Figure 4. Quantitative assessment of geniculo-cortical topography. In each graph, the error bars depict the SEM. $A$, Percentage dLGN labeled by a single injection. For coronal section, we measured the area that contained at least $10 \%$ of the peak density in a given dLGN. The summed areas were expressed as a percentage of the total area of the dLGN sections. This analysis gives an estimate of the scatter of back label throughout the dLGN, which is very high at P2 and then falls dramatically in the following week. These changes with age are highly significant (one-way ANOVA, $p<0.0005$ ). $B$, Nearest neighbor analysis. To quantify the segregation in the projection, we estimated the probability that a labeled cell has as its nearest neighbor one of the same color. A value of

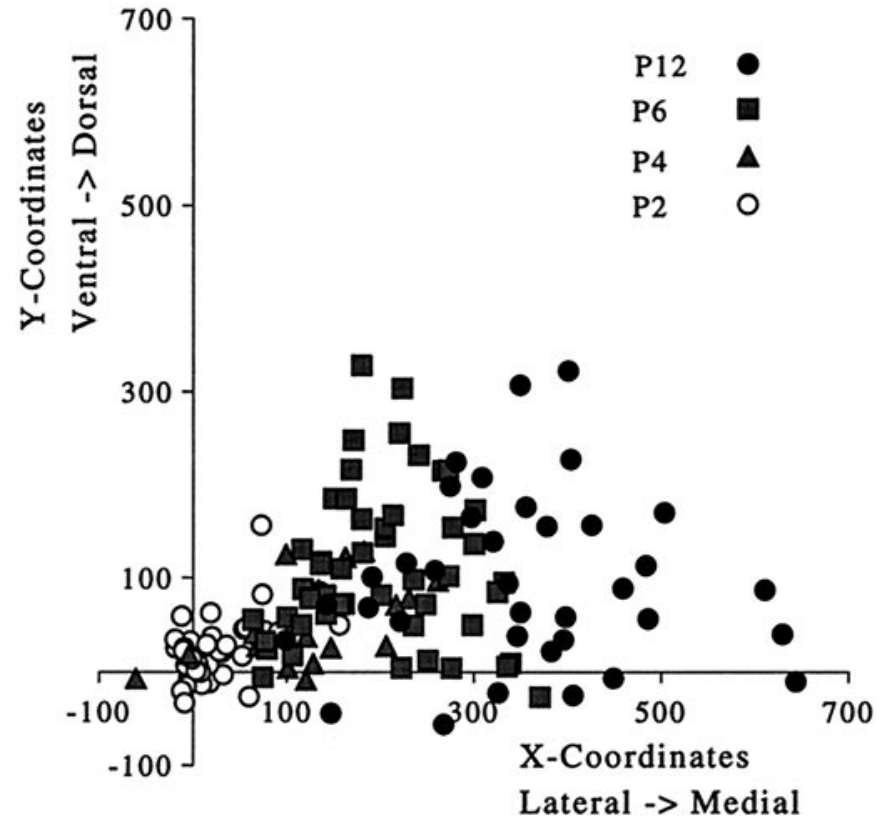

Figure 5. Vector analysis confirms early disorder. This graph describes the spatial relationship between the two populations of label in each section. Brain sections were aligned along their dorsoventral and mediolateral axes. The vector was calculated from the center of gravity of the population of cells labeled by the more medial cortical injection to the population labeled by the lateral injection. In the adult normal dLGN, this vector goes lateral to medial (Dursteler et al., 1979). Except for the P2 vectors, almost all point medially as predicted. The result at $\mathrm{P} 2$ strongly indicates no overall bias toward the adult map in the early scatter. The scatter along the dorsoventral axis has three possible causes. One reason lies in the curvature of the dLGN, which affects the alignment of the projection columns, producing some separation in the dorsoventral axis (compare Fig. 2, P6). Second, the dorsoventral and mediolateral axes of the sections are only approximately aligned. Finally, the scatter could be caused by a slight anteroposterior offset of the paired injections.

ance, the percentage of dLGN labeled by the early injection was still comparable to the value obtained at P2 (Fig. 6). It appears that inappropriately targeted cells do not die selectively to yield the adult projection. A change in the distribution of terminals can be demonstrated directly from the later placement, at P6 or P12, of a second tracer at the same cortical site. A green injection site of the same size as the earlier red placement, but made at P12, back-labeled only a tight and restricted focus of cells in the dLGN (Fig. 2), comparable to a normal P12 injection. For P6 heterochronic injections, the result is similar.

Many of the cells labeled with the second tracer were also double-labeled with the first tracer. These neurons therefore made a topographically appropriate projection at $\mathrm{P} 2$ as well as at P12. However, the fact that only a maximum of $35 \%$ of the population labeled by the second injection was double-labeled suggests that most geniculate neurons at P2 fail to make a topographically correct connection. This suggests that active terminal rearrangement rather than selective stabilization of an existing

$\sim 50 \%$, as we see at $\mathrm{P} 2$, suggests that there is no segregation of the input to the two discrete injection sites. As the connection gets more ordered, the value rises significantly (one-way ANOVA, $p<0.0005$ ). $C$, Double label. The figure displays the percentage of cells double-labeled (with respect to the smaller of the two populations) against postnatal age. This measure stays fairly constant. 
Figure 6. Percentage of dLGN labeled by a single injection, after short- or long-term survival. The graph shows what percentage of the dLGN is retrogradely labeled by a single injection into area 17 after varying survival times. The error bars represent the SEM. The three light gray bars describe the results for single injections placed at $\mathrm{P} 2, \mathrm{P} 6$, or $\mathrm{P} 12$, respectively; in these cases the animals were killed within the next $24-48 \mathrm{hr}$ ( $n=6$ in each age group). The decrease in labeled area with the maturation of the projection can be clearly seen. However, if after a P2 injection animals were allowed to grow up to P6 or P12, the percentage of dLGN labeled still resembled the P2 result and not those animals that received injections at later times (black columns; $n=$ 7 in each group). Thus, most early-labeled cells can survive the ordering of the projection, even if they did not make appropriate connections in early development.

collateral alone drives the formation of geniculo-cortical topography.

\section{DISCUSSION}

Our results show that geniculo-cortical topography is highly disordered at a time when thalamic axons are restricted to the deep cortical layers. When most axon terminals are confined to layer IV at P6, the basic map is in place. Long-term survival experiments exclude selective cell death as a major factor in the formation of the topography. A combination of collateral withdrawal and axonal targeting is implicated in map formation.

\section{Assessing order of geniculo-cortical topography}

Qualitatively and quantitatively, the early geniculo-cortical projection pattern in the hamster is disordered. A restricted injection into cortex on P2 labels cells throughout the dLGN, whereas a similar injection only 4 d later produces a well-defined column of back label. This observation and the controls illustrated in Figure 3 strongly suggest that the early disorder cannot simply be attributed to a technical artifact such as uptake by fibers of passage. The use of paired injections, with low levels of double label, also eliminates cortical spread of tracer as a cause of the scatter. Uptake of label could occur by axons growing through or extending collaterals into the injection sites. In either case, our results demonstrate that axons from throughout the dLGN contact a single cortical locus. Moreover, paired injections $500 \mu \mathrm{m}$ apart, at loci that do not even lie along the general line of thalamic ingrowth, label spatially congruent but different populations of neurons in the dLGN. These observations show that the initial topographic distribution of geniculate axons, or their collaterals, in the subplate and deepest cortical layers is extremely imprecise.

The lack of order in the early projection is confirmed by the quantitative analysis. The percentage of dLGN labeled gives an estimate of the scatter in the projection, whereas the nearest neighbor and vector analyses reflect the segregation of the geniculate input to discrete cortical points separated by an average distance of $\sim 500 \mu \mathrm{m}$. The density analysis suggests that there is some minor bias in the projection, because only $70 \%$ of the dLGN contains labeled cells at the criterion density. However, both the nearest neighbor result and the vector analysis reveal that thalamic afferents at P2 do not distinguish between two cortical sites separated by one-third the width of striate cortex. Our analyses do show that there is some refinement in topography after the map has emerged at P6 and leave open the possibility of further subtle changes after P12.

\section{Where is geniculo-cortical topography generated?}

Our results might seem to conflict with studies on the development of topography in rodent somatosensory cortex. In mouse barrel cortex, thalamic afferents display a thalamotopic map at birth, when most fibers are still confined to the lower cortical layers (Agmon et al., 1993, 1995). Adult-like barreloid topography is seen at P2 when the thalamic fibers are established in layer IV. Our results also show a topographic map when the geniculate projection has terminated in layer IV, at P6, but we find an absence of topography earlier when thalamic afferents are confined to the subplate and lower cortical layers. In their retrograde tracing at P0, however, Agmon et al. (1995) were careful to place the DiI in the dense cortical plate. This ensured that they labeled only the thalamic axons advancing through layer $\mathrm{V}$ toward layer IV and avoided the tangential plexus of fibers in layer VI (Agmon et al., 1993). Catalano et al. $(1991,1996)$ also described tangentially running, as well as locally branching, thalamic axons in the subplate (layer VIb) and layer VI in prenatal and neonatal rat somatosensory cortex. Such morphology implies topographic disorder, although retrograde tracing by Catalano et al. (1996) indicates that the disorder is local. We have concentrated on quantifying the precision of the thalamocortical map at a time when the bulk of the thalamic afferents are confined to subplate and the deepest cortical layers. Our results show that, at least for the geniculo-cortical system, the initial guidance of thalamic axons to cortex does not generate the adult map, and they imply that thalamic axons have to actively sort out in the deepest layers of the cortex, before they target layer IV. It would be interesting to establish whether paired bead injections into somatosensory cortex at a comparable developmental stage would reveal similar errors in early targeting.

Our results also have implications for the morphology of the thalamic axons. If big, overlapping terminal arbors were responsible for disorder in the projection, we would expect a high percentage of double-labeled cells and some bias in the distribution of red and green cells. We find a small but consistent percentage of double-labeled cells. This could arise if individual axons had small, scattered collateral branches and thus sampled a wide area of striate cortex (a pattern that would also contribute to the topographic disorder). Such an axonal morphology was described in the newborn hamster by Naegele et al. (1988) after labeling single thalamic terminal arbors from HRP injections into the optic radiation. Between $\mathrm{P} 0$ and $\mathrm{P} 2$, axons extended multiple short collaterals into subplate and lower regions of the cortical 
plate. It would be interesting to know what role these early collaterals play in the guidance of thalamic axons to their final targets.

\section{A role for cell death?}

The fact that the diffuse distribution of geniculate neurons labeled early in development persists into the second postnatal week eliminates selective cell death as the main mechanism generating geniculo-cortical topography. The heterochronic double-tracer injections also suggest that the map is not generated simply by the stabilization of a topographically appropriate collateral. In this case, we would expect that all cells labeled by the second injection should also be labeled by the first injection. The fact that there is a relatively low rate of double labeling with heterochronic injections indicates considerable terminal rearrangement during cortical map formation. Thalamic cell death does occur in postnatal rodent. In the ventrobasal nucleus of the rat, over one-quarter of all cells die during the first postnatal week, with a peak at the day of birth (Waite et al., 1992). Pyknotic cell counts in postnatal hamster dLGN indicate peaks of cell death around P5 and P8, particularly near the periphery of the nucleus (Sengelaub et al., 1985). Although a minor contribution of cell death to the establishment of topography might be possible, our observations make it seem more likely that cell death is involved in size matching rather than in the generation of the geniculo-cortical map.

\section{Implications for map formation in the cortex}

An exchange of neighbors in the thalamocortical projection is a necessary requirement for primary sensory map formation (Connolly and Van Essen, 1984; Nelson and LeVay, 1985; Adams et al., 1997). Nelson and LeVay (1985) suggested that the rearrangement of geniculate fibers takes place in the white matter underlying visual cortex. Our results strongly implicate the cortical subplate in map formation and therefore in the exchange of neighbors. They also leave little room for ordered ingrowth of the geniculate afferents or reciprocal guiding of geniculo-cortical and cortico-geniculate fibers in forming the topographic map in area 17 , although the internal order in the fiber bundle could be well preserved up to the subplate. Despite disorder within the projection, our anterograde and retrograde tracing suggest accurate targeting of area 17 as a whole by the thalamic axons from the dLGN. It is possible that quite different principles guide the accurate targeting of thalamus to a given cortical area and the generation of topographic order within that area. For instance, it has been suggested that axons from different parts of the thalamus fasciculate in distinct bundles and do not mix on their way to their cortical targets (Blakemore and Molnar, 1990). Indeed, the mapping of the whole thalamus onto neocortex could be achieved by ordered ingrowth, because there is no topological mismatch between these structures as a whole (Adams et al., 1997).

What mechanisms might guide the formation of cortical topography from the early disordered input we have shown? The balance between activity-independent and activity-dependent mechanisms needs to be investigated. In the retinocollicular system of the rodent, both mechanisms appear to contribute to the generation of the retinotopic map. Molecular gradients could lay out the basic polarity of a crude map, whereas Hebbian synapses would underlie the refinement of topography (Walter et al., 1987; Nakamoto et al., 1996; Simon et al., 1992). It is not known whether molecular gradients exist within cortical areas, but certainly neural activity has been implicated in both the refinement of the basic somatosensory thalamocortical map (Fox et al., 1996), and the initial arborization of the geniculate afferents in cat visual cortex (Herrmann and Shatz, 1995). The machinery for map refinement via neural activity appears to be present in the immature visual system. During the early development of visual pathways, waves of patterned spontaneous activity have been demonstrated in the rodent and carnivore retina (Maffei and Galli-Resta, 1990; Meister et al., 1991; Wong et al., 1993). This activity can be transmitted through the dLGN (Mooney et al., 1996) and could potentially activate subplate cells (Friauf et al., 1990), providing a substrate for Hebbian refinement. It will be interesting to see how activity-dependent and -independent mechanisms are balanced in the development of the geniculocortical map, a map in which order emerges from disorder.

\section{REFERENCES}

Adams NC, Lozsadi LD, Guillery RW (1997) Complexities in the thalamocortical and corticothalamic pathways. Eur J Neurosci 9:204-209.

Agmon A, Yang LT, O’Dowd DK, Jones EG (1993) Organized growth of thalamocortical axons from the deep tier of terminations into layer IV of developing mouse barrel cortex. J Neurosci 13:5365-5382.

Agmon A, Yang LT, Jones EG, O’Dowd DK (1995) Topological precision in the thalamic projection to neonatal mouse barrel cortex. J Neurosci 15:549-561.

Blakemore C, Molnar Z (1990) Factors involved in the establishment of specific interconnections between thalamus and cerebral cortex. Cold Spring Harb Symp Quant Biol 55:491-504.

Catalano SM, Robertson RT, Killackey HP (1991) Early ingrowth of thalamocortical afferents to the neocortex of the prenatal rat. Proc Natl Acad Sci USA 88:2999-3003.

Catalano SM, Robertson RT, Killackey HP (1996) Individual axon morphology and thalamocortical topography in developing rat somatosensory cortex. J Comp Neurol 366:36-53.

Chalupa LM, Snider CJ, Kirby MA (1996) Topographic organization in the retinocollicular pathway of the fetal cat demonstrated by retrograde labeling of ganglion cells. J Comp Neurol 368:295-303.

Connolly M, Van Essen D (1984) The representation of the visual field in parvicellular and magnocellular layers of the lateral geniculate nucleus in the macaque monkey. J Comp Neurol 226:544-564.

Crossland WJ, Uchwat CJ (1982) Neurogenesis in the central visual pathways of the golden hamster. Brain Res 281:99-103.

Dursteler MR, Blakemore C, Garey LJ (1979) Projections to the visual cortex in the golden hamster. J Comp Neurol 183:185-204.

Fox K, Schlaggar BL, Glazewski S, O'Leary DD (1996) Glutamate receptor blockade at cortical synapses disrupts development of thalamocortical and columnar organization in somatosensory cortex. Proc Natl Acad Sci USA 93:5584-5589.

Friauf E, McConnell SK, Shatz CJ (1990) Functional synaptic circuits in the subplate during fetal and early postnatal development of cat visual cortex. J Neurosci 10:2601-2613.

Herrmann K, Shatz CJ (1995) Blockade of action potential activity alters initial arborization of thalamic axons within cortical layer 4. Proc Natl Acad Sci USA 92:11244-11248.

Krug K, Thompson ID (1996) Mechanisms involved in the normal and abnormal development of geniculo-cortical topography in the Syrian hamster. Soc Neurosci Abstr 22:1016.

Krug K, Trevelyan AJ, Thompson ID (1995) A study of abnormal geniculo-cortical topography in the anaesthetised Syrian hamster. J Physiol (Lond) 485:9P.

Maffei L, Galli-Resta L (1990) Correlation in the discharges of neighboring rat retinal ganglion cells during prenatal life. Proc Natl Acad Sci USA 87:2861-2864.

Meister M, Wong RO, Baylor DA, Shatz CJ Meister (1991) Synchronous bursts of action potentials in ganglion cells of the developing mammalian retina. Science 252:939-943.

Mesulam MM (1982) Principles of horseradish peroxidase neurohistochemistry and their applications for tracing neural pathways. In: Tracing neural connections with horseradish peroxidase (Mesulam MM, ed), pp 1-151. Chichester, UK: Wiley.

Miller B, Chou L, Finlay BL (1993) The early development of thalamocortical and corticothalamic projections. J Comp Neurol 335:16-41. 
Mooney R, Penn AA, Gallego R, Shatz CJ (1996) Thalamic relay of spontaneous retinal activity prior to vision. Neuron 17:863-874.

Naegele JR, Jhaveri S, Schneider GE (1988) Sharpening of topographical projections and maturation of geniculocortical axon arbors in the hamster. J Comp Neurol 277:593-607.

Nakamoto M, Cheng HJ, Friedman GC, McLaughlin T, Hansen MJ, Yoon CH, O'Leary DD, Flanagan JG (1996) Topographically specific effects of ELF-1 on retinal axon guidance in vitro and retinal axon mapping in vivo. Cell 86:755-766.

Nelson SB, LeVay S (1985) Topographic organization of the optic radiation of the cat. J Comp Neurol 240:322-330.

O'Leary DD, Fawcett JW, Cowan WM (1986) Topographic targeting errors in the retinocollicular projection and their elimination by selective ganglion cell death. J Neurosci 6:3692-3705.

Reese BE, Baker GE (1993) The re-establishment of the representation of the dorso-ventral axis in the chiasmatic region of the ferret. Vis Neurosci 10:957-968.

Scholes JH (1979) Nerve fibre topography in the retinal projection to the tectum. Nature 278:620-624.

Sengelaub DR, Jacobs LF, Finlay BL (1985) Regional differences in normally occurring cell death in the developing hamster lateral geniculate nuclei. Neurosci Lett 55:103-108.

Simon DK, O'Leary DD (1991) Relationship of retinotopic ordering of axons in the optic pathway to the formation of visual maps in central targets. J Comp Neurol 307:393-404.
Simon DK, O’Leary DD (1992) Development of topographic order in the mammalian retinocollicular projection. J Neurosci 12:1212-1232.

Simon DK, Prusky GT, O'Leary DD, Constantine Paton M (1992) $\mathrm{N}$-methyl-D-aspartate receptor antagonists disrupt the formation of a mammalian neural map. Proc Natl Acad Sci USA 89:10593-10597.

Smith AL, Krug K, Thompson ID (1994) The development of normal and abnormal geniculo-cortical topographies in the Syrian hamster. Soc Neurosci Abstr 20:1704.

Torrealba F, Guillery RW, Eysel U, Polley EH, Mason CA (1982) Studies of retinal representations within the cat's optic tract. J Comp Neurol 211:377-396.

Trevelyan AJ (1992) Development of the mammalian geniculo-cortical projection. $\mathrm{PhD}$ thesis, Oxford University.

Trevelyan AJ, Thompson ID (1992) Altered topography in the geniculocortical projection of the golden hamster following neonatal monocular enucleation. Eur J Neurosci 4:1104-1111.

Waite PM, Li L, Ashwell KW (1992) Developmental and lesion induced cell death in the rat ventrobasal complex. NeuroReport 3:485-488.

Walter J, Henke-Fahle S, Bonhoeffer F (1987) Avoidance of posterior tectal membranes by temporal retinal axons. Development 101:909-913.

Wong RO, Meister M, Shatz CJ (1993) Transient period of correlated bursting activity during development of the mammalian retina. Neuron 11:923-938. 